Check for updates

Cite this: RSC Adv., 2019, 9, 2244

Received 9th October 2018

Accepted 31st December 2018

DOI: $10.1039 / \mathrm{c} 8 \mathrm{ra0} 0363 \mathrm{k}$

rsc.li/rsc-advances

\section{Electrospun composite nanofibre fabrics containing green reduced Ag nanoparticles as an innovative type of antimicrobial insole}

\begin{abstract}
L. Du, (D) ${ }^{a b}$ T. Li, $^{a}$ S. Wu, ${ }^{a}$ H. F. Zhu ${ }^{\text {ab }}$ and F. Y. Zou (D)*ab
In this study, polyvinyl alcohol (PVA) nanofibrous membranes containing silver nanoparticles (Ag NPs) were successfully fabricated by the combination of electrospinning and a green reduction approach. Through the electrospinning technique, uniform and smooth nanofibres can be obtained, and the Ag NPs with a narrow size distributions are well dispersed in PVA nanofibres. The investigation indicates that the mass ratio of reductant tea polyphenols and $\mathrm{AgNO}_{3}$ play a crucial role in controlling the size of the Ag NPs. More importantly, multi-layered fabrics with a layer of PVA/Ag NP nanofibrous membrane layered onto cotton substrates were developed and applied to shoe insoles. The fabricated shoe insoles with functionalized PVA nanofibres exhibit remarkable antimicrobial activity against both E. coli and S. aureus (i.e. antibacterial rate $>99 \%$ ). The creation of such an encouraging fabric could establish a new optimization methodology for producing nanoengineered functional textiles.
\end{abstract}

\section{Introduction}

Textiles have long been recognized as media that provide a suitable substrate for the growth of microorganisms owing to the appropriate humidity and temperature. ${ }^{1}$ Especially, shoe insoles are vulnerable to be contaminated by microorganisms in wearing because of the presence of the high moisture and warmth conditions, nutrients from feet sweat and greases. ${ }^{2,3}$ The microorganisms' growth in the enclosed environment of the inner shoe may cause athlete's foot or undesired odours. Thus, it is necessary to impart an antimicrobial function into insoles to inhibit the growth of microbes. ${ }^{4}$

Silver has been most widely recognized for its application in medical and other scientific fields, on account of its acknowledged bactericidal effect. ${ }^{5}$ However, recent enhancements have been realized using silver nanoparticles (Ag NPs) with an attractive anti-microbial activity, which can be obtained through physical, chemical, and biological modes of reduction..$^{6-8}$ In particular, environmentally friendly green reductants that assist the biomimetic synthesis of Ag NPs have spurred numerous investigations. ${ }^{9}$ The green synthesis of Ag NPs using microorganisms and plant extracts has received a great deal of attention, the latter one being the best alternative to obtain Ag NPs due to the ease and efficacy in the reduction of silver ions by the biomolecules present in plant extracts. ${ }^{10}$ In the present

${ }^{a}$ School of Fashion Design \& Engineering, Zhejiang Sci-Tech University, Hangzhou 310018, China

${ }^{b}$ Zhejiang Provincial Research Center of Clothing Engineering Technology, Zhejiang Sci-Tech University, Hangzhou 310018, China. E-mail: zfy166@zstu.edu.cn; Fax: $+86-571-86843249$ study, we proposed a facile and green approach to synthesize Ag NPs, selecting tea polyphenols (TP) as the reductant. TP extracted from tea plants is a water-soluble polyphenol compound belonging to the flavonoid family, mainly consisting of epicatechin (EC), epicatechin gallate (ECG), epigallocatechin (EGC) and epigallocatechin gallate (EGCG). Of these, EGCG accounts for $50-60 \%$ of the total TPs. Meanwhile, such a compound exhibits highly water-soluble and excellent antioxidant properties due to its multiple pyrogallol and catechol groups..$^{11,12}$

It is also worth noting that naked Ag NPs are easily oxidized during storage and tend to aggregate due to the high surface energy, resulting in a remarkable reduction in their antibacterial activity. ${ }^{13}$ Thus, it is of utmost necessity to maintain stability and antibacterial activity by incorporating Ag NPs with biodegradable polymers. Polyvinyl alcohol (PVA), a well-known watersoluble polymer with good biocompatibility, has been widely used in several biomedical applications, like soft contact lenses, wound dressing, and tissue engineering. ${ }^{14-16}$ Therefore, in view of the excellent characteristic of PVA, we prepared monodispersed Ag NPs which were embedded into the PVA nanofibres.

Electrospinning is a sophisticated technique for the production of ultrafine fibres or fibrous membranes with diameters in the nanometre to micron range. The electrospun nanofibrous membranes exhibit attractive properties, such as high specific surface area, high porosity and flexibility. ${ }^{17,18}$ Also, the nanofibrous membranes can be easily functionalized through incorporating an appropriate agent or deliberately designing and controlling (manipulating) the structure of the electrospun fibre. ${ }^{19}$ More recently, there has been growing 
consumer demand for durable and functional apparel manufactured in a sustainable manner, implicating an opportunity for electrospun nanofibrous membranes to be integrated into textile substrates. ${ }^{20}$ For instance, in virtue of electrospinning and a doctor-blading coating modification, $\mathrm{Xu}$ et al. fabricated superhydrophobic polyacrylonitrile/polyurethane nanofibrous membranes which contain titanium dioxide nanoparticles, with characteristics such as UV resistance, water repellency and moisture breathability. ${ }^{21}$ Babar et al. prepared colourful cellulose acetate-based nanofibrous mats with exceptional directional moisture transport performance. ${ }^{22}$ Their findings all indicate that electrospun nanofibrous membranes integrated into textiles will be an effective and necessary strategy to provide multiple functionalities into high-value-added textiles.

Herein, a convenient method of introducing TP as the green reductant to biosynthesize Ag NPs is proposed through an in situ reduction approach in PVA aqueous solutions. Also, different crosslinking conditions for PVA/Ag NP nanofibrous membranes were explored, which can guarantee a fibre morphology and a fibrous mat pore structure after immersing in water for a certain period of time. Meanwhile, we report a facile strategy for the fabrication of electrospun composite nanofibre fabrics as an antimicrobial insole. The insole consisted of a PVA/Ag NP nanofibrous membrane as an interlayer, a two-sided fusible interlining as the adhesive layer, and woven cotton as the substrate. The mechanical property and antibacterial activity of the insoles were evaluated.

\section{Materials and methods}

\subsection{Materials}

Poly(vinyl alcohol) (PVA) powders (99\% hydrolyzed, $M_{\mathrm{w}}=$ $\left.145000 \mathrm{~g} \mathrm{~mol}^{-1}\right)$ and silver nitrate $\left(\mathrm{AgNO}_{3}\right.$, purity $\left.99.8 \%\right)$ were obtained from Aladdin Chemistry Co., Ltd., China. Tea polyphenols (TP, purity 98\%) were acquired from Shanghai Jinsui Biotechnology Co., Ltd., China. Glutaraldehyde solutions (GA, $25 \% \mathrm{v} / \mathrm{v}$ in water, and $50 \% \mathrm{v} / \mathrm{v}$ in water) were purchased from Kermel Chemical Reagent Co., Ltd., China. Nutrient Agar (containing agar powder, peptone, beef extract powder, and sodium chloride) was purchased from Hangzhou Baisi Biotechnology Co., Ltd., China. The Staphylococcus aureus (S. aureus, ATCC 6538) as the Gram-positive and Escherichia coli (E. coli, ATCC 8099) as the Gram-negative model bacteria were bought from Taojunzhu Biotechnology Co. Ltd., China. Phosphate buffer saline (PBS, $0.03 \mathrm{M}, \mathrm{pH}=7.4$ ) was obtained from Phygene Life Sciences Co., Ltd., China. Deionized water (DIW) with a resistance of $c a .18 \mathrm{M} \Omega \mathrm{cm}$ was used in the experiment.

\subsection{Preparation of solution}

The $\mathrm{AgNO}_{3}$ aqueous solutions with TPs were prepared according to our previous work. ${ }^{11}$ The electrospun PVA solution containing green reduced Ag NPs was prepared via three steps: firstly, the PVA aqueous solution with a concentration of $10 \mathrm{wt} \%$ was prepared by vigorous stirring for $2 \mathrm{~h}$ at $80^{\circ} \mathrm{C}$. Then, the $2 \mathrm{wt} \% \mathrm{AgNO}_{3}$ (relative to the weight of PVA) was dissolved in the PVA solution, and the mixture was kept uninterruptedly stirred for $0.5 \mathrm{~h}$ when the solution temperature lowered to $65{ }^{\circ} \mathrm{C}$. Finally, $60 \mathrm{wt} \% \mathrm{TP}$ (relative to the weight of $\mathrm{AgNO}_{3}$ ) was added dropwise, and sufficient stirring for $2 \mathrm{~h}$ was imposed in a dark environment.

\subsection{Preparation of membranes}

To prepare the PVA nanofibrous membrane containing Ag NPs, an electrospinning setup composed of a high voltage power supply (0-50 kV, DE-100, Ding Tong, China), a rotating drum collector, and a syringe pump (TYD 01-01, Lei Fu, China) equipped with a $10 \mathrm{~mL}$ syringe (with a $21 \mathrm{G}$ stainless steel needle and without rubber) was used. The parameters were set as follows: a feed rate of $8 \mu \mathrm{L} \mathrm{min}{ }^{-1}$, a tip-to-collector distance of $15 \mathrm{~cm}$, an applied voltage of $12 \mathrm{kV}$, and a roller speed of $200 \mathrm{rpm}$. The temperature and relative humidity (RH) were regulated and kept at $25 \pm 2{ }^{\circ} \mathrm{C}$ and $32 \pm 3 \%$, respectively. To obtain PVA/Ag NP nanofibrous membrane with water stability, the samples should be crosslinked in glutaraldehyde vapor (GA) in a vacuum chamber. Also, the effects of cross-linking conditions (i.e. agent concentration, cross-linking temperature and cross-linking time) on the membrane performance were systematically investigated.

\subsection{Preparation of shoe insole}

The antimicrobial shoe insoles with a sandwich structure consist of five layers, i.e., woven cotton as the substrate, twosided fusible interlining as the adhesive layer, and a PVA/Ag NP nanofibrous membrane as the functional interlayer. The control specimens were prepared with a similar structure, in which the neat PVA nanofibrous membrane is selected as the functional interlayer. A schematic diagram of the shoe insoles is shown in Fig. 1.

\subsection{Characterization and measurements}

To confirm the reducibility of TP, we measured the change of the $\mathrm{AgNO}_{3} / \mathrm{TP}$ aqueous solution spectra and conductivity by ultraviolet-visible spectroscopy (UV-vis, Lambda 35, PerkinElmer, USA) and an electrical conductivity meter (DDSJ-308A, Inesa \& Scientific Instruments Co., China). Fourier transform infrared spectroscopy (FT-IR) was performed using a Nicolet 5700 spectrometer (Thermo Electron Corporation, USA).

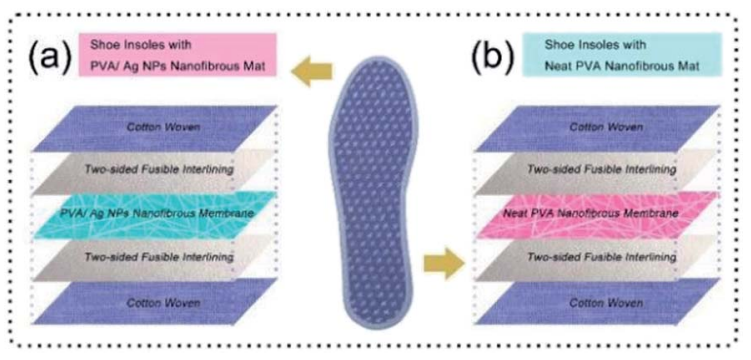

Fig. 1 Schematic illustration of the shoe insoles: (a) shoe insoles with PVA/Ag NP nanofibrous mat; (b) shoe insoles with neat PVA nanofibrous mat. 

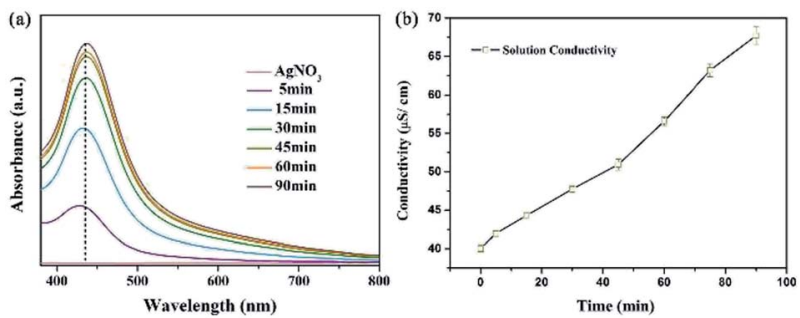

Fig. 2 (a) UV-vis spectra of Ag NPs in aqueous solution; (b) the change of the reaction solution conductivity.

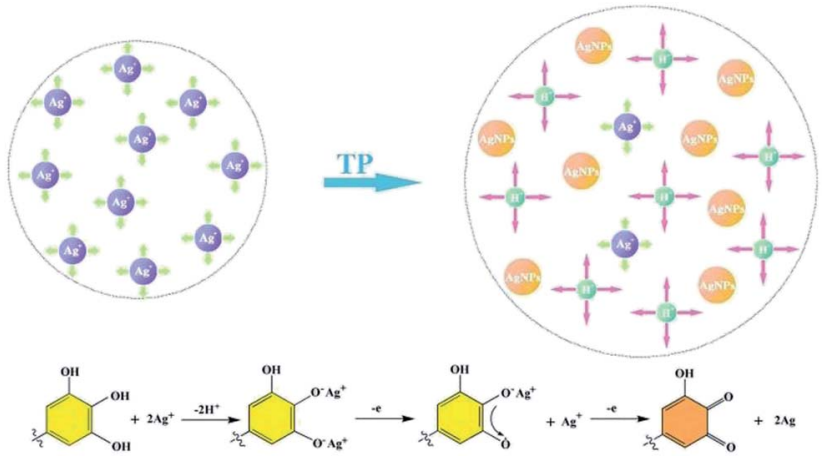

Fig. 3 Schematic illustration of the mechanism for the change of the Ag NP aqueous solution conductivity.

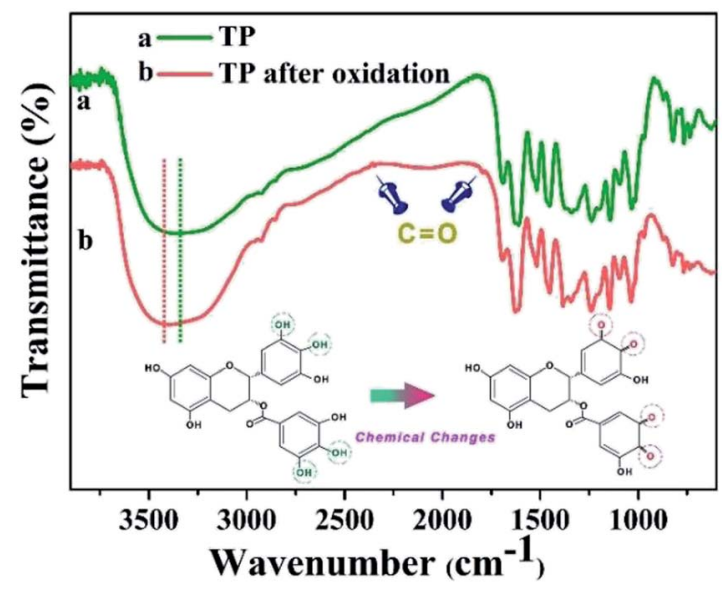

Fig. 4 The FTIR spectra of (a) TP and (b) TP after oxidation.
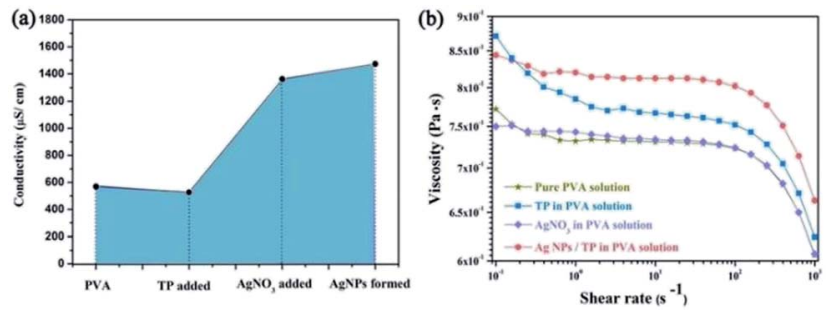

Fig. 5 (a) Conductivities of electrospun precursor solutions; (b) viscosities of electrospun precursor solutions.

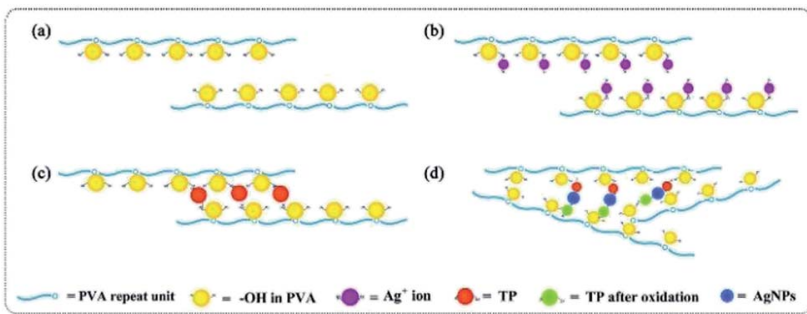

Fig. 6 Schematic illustration of the mechanism for the changes of the solution viscosities.

Furthermore, conductivities and viscosities of polymer solutions were measured before the electrospinning. The viscosity data were obtained through a rheometer (MCR 301, Anton Paar $\mathrm{GmbH}$, Austria), whose shear rate ranges from 0.1 to $1000 \mathrm{~s}^{-1}$ with a double crack coaxial cylinder (DG-26.7). The solution conductivities were achieved via a conductivity meter.

The surface morphology of the fibrous membranes was examined by field emission scanning electron microscopy (FESEM, vltra55, Carl Zeiss, Germany) at $3.0 \mathrm{kV}$. The diameters of the nanofibres were determined from the FE-SEM image using the Image Pro-Plus software (200 fibres of were randomly selected for the measurement). The distributions of Ag NPs in the nanofibres were acquired via Transmission Electron Microscopy (TEM, JSM-2100, JEOL, Japan) at an accelerating voltage of $200 \mathrm{kV}$. The crystalline structure of the samples was determined by X-ray Diffraction (XRD, D8 discover, Bruker, Germany) with $\mathrm{Cu} \mathrm{K}_{\alpha}$ radiation $(\lambda=1.5406 \AA)$ in the range of 10 $90^{\circ}$ at a scan speed of $3^{\circ} \mathrm{min}^{-1}$.

The mechanical properties of nanofibrous membranes (i.e. neat PVA and PVA/Ag NPs) were tested using a multifunctional material tensile tester (KES-G1, KATO, Japan). Specimens were cut at $5 \mathrm{~mm}$ width and $40 \mathrm{~mm}$ length and run in the instrument with a constant speed of $12 \mathrm{~mm} \mathrm{~min}{ }^{-1}$ using standard procedures (ASTM standard 882 for thin films and membranes). The mechanical property of the shoe insoles with neat PVA and shoe insoles with PVA/Ag NP nanofibrous membrane were evaluated using a mechanical tester (Model 3344, Instron Corporation, Norwood, MA, USA) based on the ASTM standard D638. The shape of the specimens was rectangular with the size of $150 \times$ 25 square millimetres. A total of three experiments were performed on each sample group.

The antibacterial performances of shoe insoles against $E$. coli, and S. aureus were studied using a shaking flask method. A typical procedure was as follows: a mass of $0.75 \mathrm{~g}$ of the sample fabrics cut into small pieces of approximately $1 \times 1$ square centimetres was dipped into a flask containing $50 \mathrm{~mL}$ of a $0.5 \mathrm{mM}$ monopotassium phosphate (PBS) culture solution with a cell concentration of $\times 10^{5} \mathrm{CFU} \mathrm{mL}^{-1}$. The flasks were then shaken at $150 \mathrm{rpm}$ on a rotary shaker at $24^{\circ} \mathrm{C}$. Given an $18 \mathrm{~h}$ incubation, a volume of $1 \mathrm{~mL}$ of microbial suspension was taken and stepwise diluted. After incubation at $37^{\circ} \mathrm{C}$ for $24 \mathrm{~h}$, the number of microbial colonies plated on the nutrient on the agar plate was counted. The counts were used to calculate the surviving number of bacteria. The antibacterial rate of the specimens was calculated according to eqn (1), 

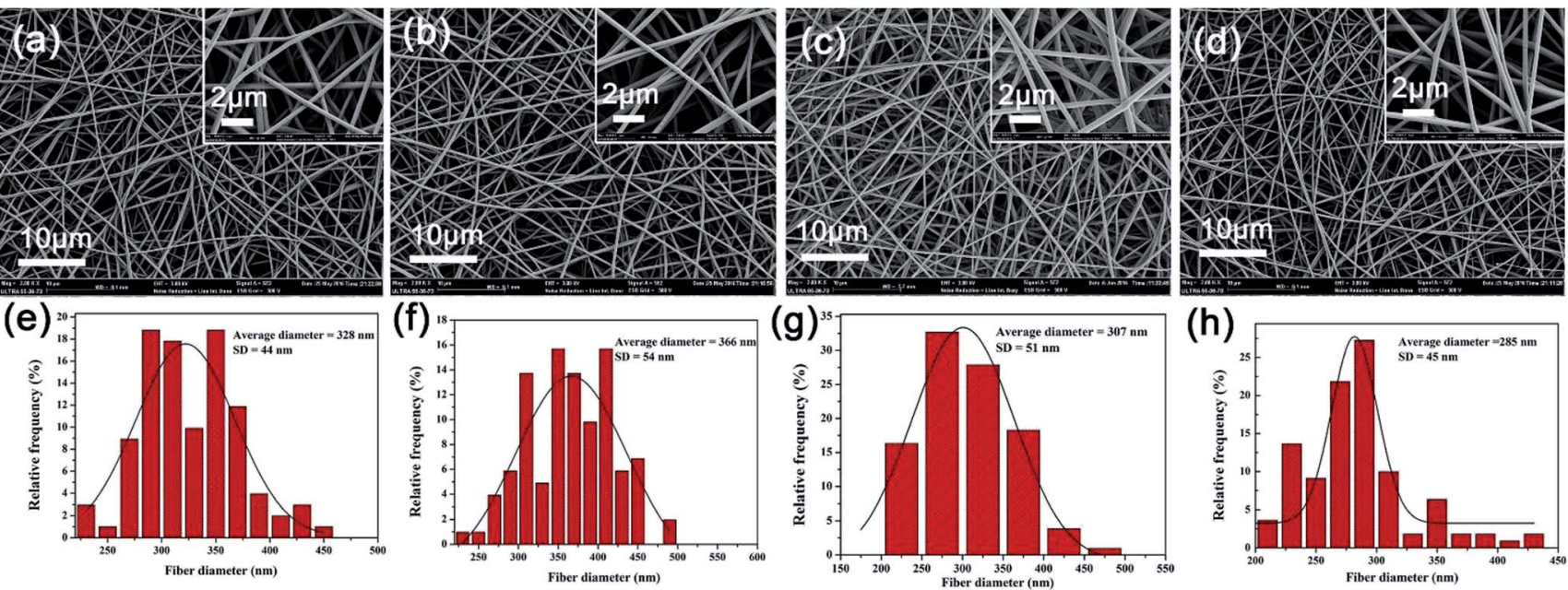

Fig. 7 SEM images of the (a) neat PVA; (b) PVA/TP; (c) PVA/AgNO ; and (d) PVA/Ag NP nanofibres. The corresponding diameter distributions of (e) neat PVA; (f) PVA/TP; (g) PVA/AgNO 3 ; and (h) PVA/Ag NP nanofibres.

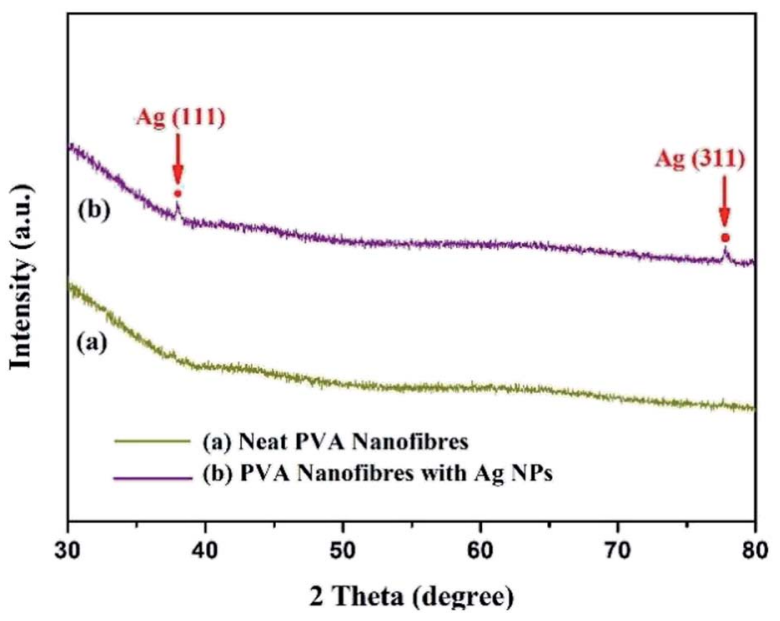

Fig. 8 The XRD pattern of the nanofibre mats: neat PVA and PVA/Ag NPS.

$$
\text { antibacterial } \operatorname{rate}(\%)=\left(1-\frac{W_{\mathrm{A}}}{W_{\mathrm{N}}}\right) \times 100
$$

where $W_{\mathrm{A}}$ and $W_{\mathrm{N}}$ stand for the numbers of viable bacterial colonies of the shoe insoles with PVA/Ag NPs and shoe insoles with neat PVA, respectively. All the experiments were repeated three times and the results are presented as mean values.

\section{Results and discussion}

The spectra and conductivity variation of the $\mathrm{AgNO}_{3}$ aqueous solutions with adding TP at different reaction times are displayed in Fig. 2. Fig. 2a shows the UV-vis spectra of the solutions. The peak at $435 \mathrm{~nm}$ in all the solutions corresponds to the surface plasmon resonance (SPR) of Ag NPs. ${ }^{23}$ Meanwhile, the conductivity of the $\mathrm{AgNO}_{3}$ aqueous solution increases greatly due to the existence of the $\mathrm{H}^{+}$ion which is formed in the reduction process of silver nanoparticles. Particularly, the $\mathrm{H}^{+}$
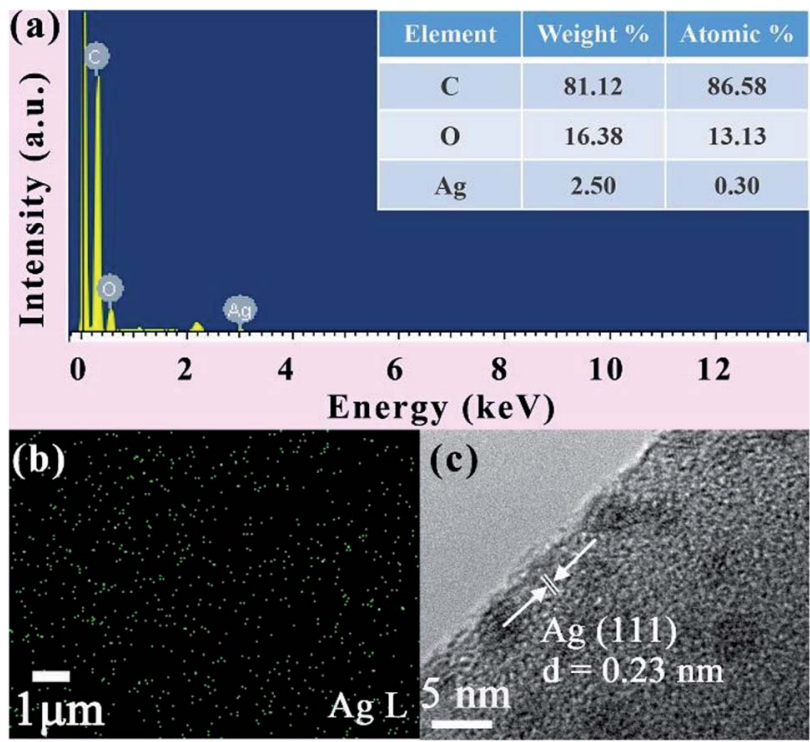

Fig. 9 (a) The EDS of PVA/Ag NP nanofibre mats; (b) elemental mapping of $\mathrm{Ag}$; (c) HRTEM image of Ag NPs.

ion has a higher ionic activity than the $\mathrm{Ag}^{+}$ions, ${ }^{24}$ which is presented as the increase of solution conductivity (see Fig. 3). The variation of spectra and conductivity indicate that TP is an excellent reductant to produce Ag NPs.

FTIR analysis was used to test the reducibility of TP toward $\mathrm{Ag}$ ions and investigate the chemical changes during the reaction process. The FTIR spectra of TP and the oxidized TP and the chemical changes before and after oxidation are indicated in Fig. 4. The absorption band at $3346 \mathrm{~cm}^{-1}$ gets narrow and shifts to $3428 \mathrm{~cm}^{-1}$, which implies the hydroxyl ions of TP are involved in the reduction of $\mathrm{Ag}^{+}$ions, resulting in the partial destruction of the hydrogen bonds of TP and an enhancement of the attracting electron effect. Based on the comparison spectrum of oxidized TP, the emerging absorption bands at $2359 \mathrm{~cm}^{-1}$ and $1792 \mathrm{~cm}^{-1}$ in the oxidized TP was ascribed to the 
(A)

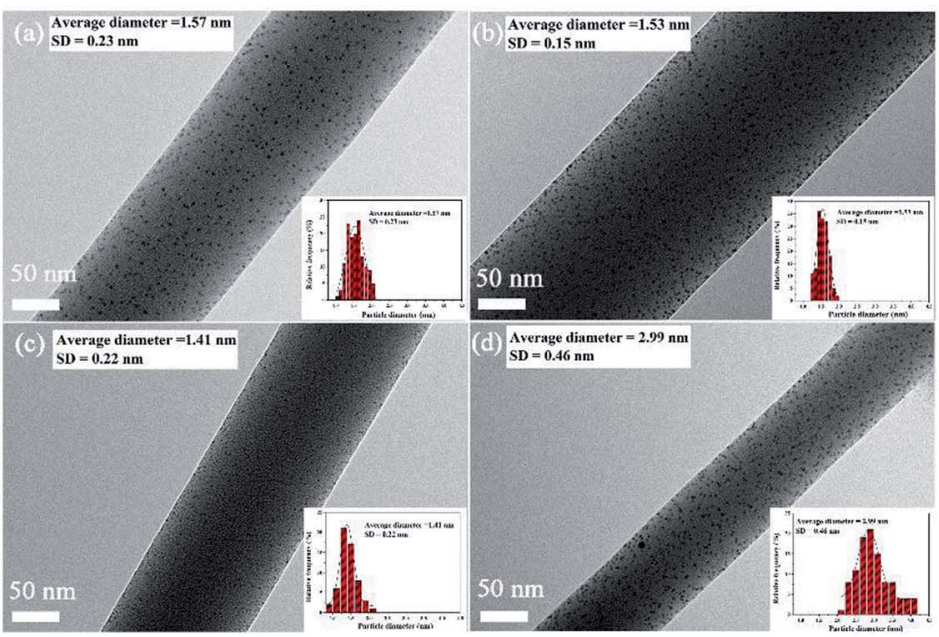

(B)

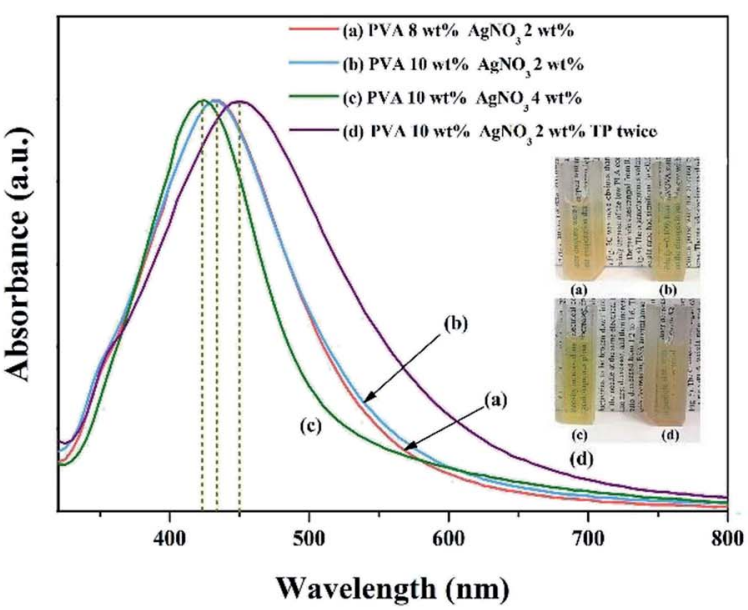

Fig. 10 (A) TEM images of the PVA/Ag NP nanofibres with different average sizes of Ag NPs by changing the solution proportion, (a) PVA 8 wt\%, $\mathrm{AgNO}_{3} 2$ wt\% (relative to the weight of PVA); (b) PVA 10 wt\%, $\mathrm{AgNO}_{3} 2 \mathrm{wt} \%$; (c) PVA $10 \mathrm{wt} \%, \mathrm{AgNO}_{3} 4 \mathrm{wt} \%$; (d) PVA $10 \mathrm{wt} \%, \mathrm{AgNO} 32 \mathrm{wt} \%$, TP dose twice. (B) UV-vis spectra of Ag NPs in the different reaction systems, and the insets are the photographs of the corresponding Ag NP aqueous solution. ${ }^{15}$

stretching vibration of $\mathrm{C}=\mathrm{O}$, suggesting the formation of the orthoquinone structure in $\mathrm{TP}^{25,26}$

Fig. 5 shows the viscosities and conductivities of electrospun precursor solutions. The pure PVA conductivity is high since the PVA is produced from poly(vinyl esters) in alkali conditions leading to some residual ions. ${ }^{27}$ There will be a decrease in the solution conductivity with the addition of TP, owing to increasing solution viscosity which may restrain ion migration. Besides, the PVA/Ag NP solution has the highest conductivity, consistent with the condition of the aqueous solution which can also be explained by the ionic activity theory. The solution viscosity has no noticeable change after the addition of $\mathrm{AgNO}_{3}$. Nevertheless, the addition of TP or the formation of Ag NPs leads an increase of solution viscosity, suggesting that interaction between TP and PVA through hydrogen bonding may help to improve the length of the PVA molecular chains. Furthermore, the latter is more obvious than the former, which can be ascribed to TP surrounding the Ag NPs leading to more opportunity for the formation of hydrogen-bonds, as exhibited in Fig. 6.

In the electrospinning process, solution properties will be one of the key factors to determine the electrospun nanofibres' morphology. ${ }^{28}$ Fig. 7 illustrates the detailed morphology of the nanofibres. Neat PVA fibrous membranes exhibited an even morphology feature with a randomly oriented 3D fibrous structure. The addition of TP resulted in a small increase in fibre diameter (from 328 to $366 \mathrm{~nm}$ ) of the resultant fibres, which can be attributed to the enhanced viscosity and decreased conductivity of the solution. The average diameter of PVA/Ag NPs (i.e. $285 \mathrm{~nm}$ ) is smaller than that of the neat PVA (i.e. $328 \mathrm{~nm}$ ), since the conductivity of the PVA/Ag NP solution is higher than that of the pure PVA solution. Regarding the effect on the electrostatic jet, a change of conductivity plays a stronger role than changes of viscosity. The higher conductivity leads to larger residual positive charges to load on the jet surface, which directly causes a greater electrostatic repulsion, and thus fibres are stretched into thinner fibres with a smaller diameter.

The crystalline phase of the nanofibres was determined by XRD. For the pattern of PVA/Ag NP mats, it is observed that diffraction peaks presented with 2 theta of $38.0^{\circ}$ and $77.3^{\circ}$, which refer to the planes of (1 111 ) and (3 111 ) for the FCC phase $\mathrm{Ag}$ (JCPDS card no. 04-0783) ${ }^{29}$ (see Fig. 8). The energy-dispersive X-ray spectroscopy (EDX), corresponding elemental mapping and high resolution TEM (HR-TEM) image further confirm the presence of Ag, and are displayed in Fig. 9. The lattice fringes can be clearly seen from the HRTEM image of Ag NPs. And the

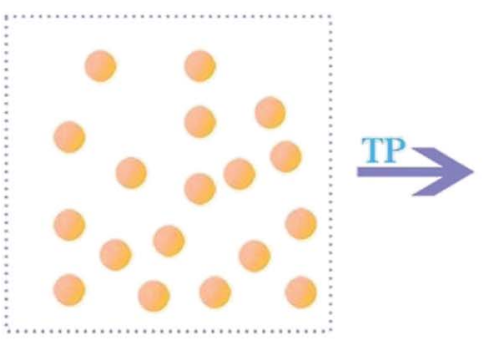

Generation of Atoms

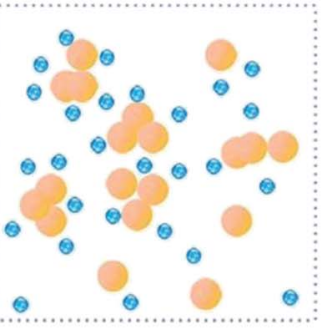

Self-nucleation

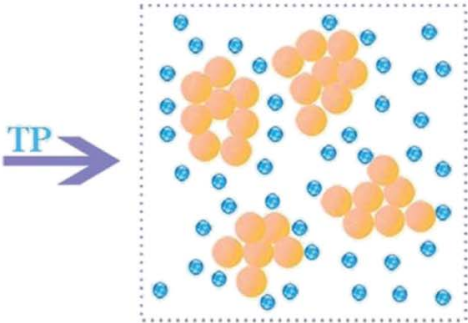

Growth

Fig. 11 Schematic illustration of the mechanism for the nucleation and growth of Ag NPs. 

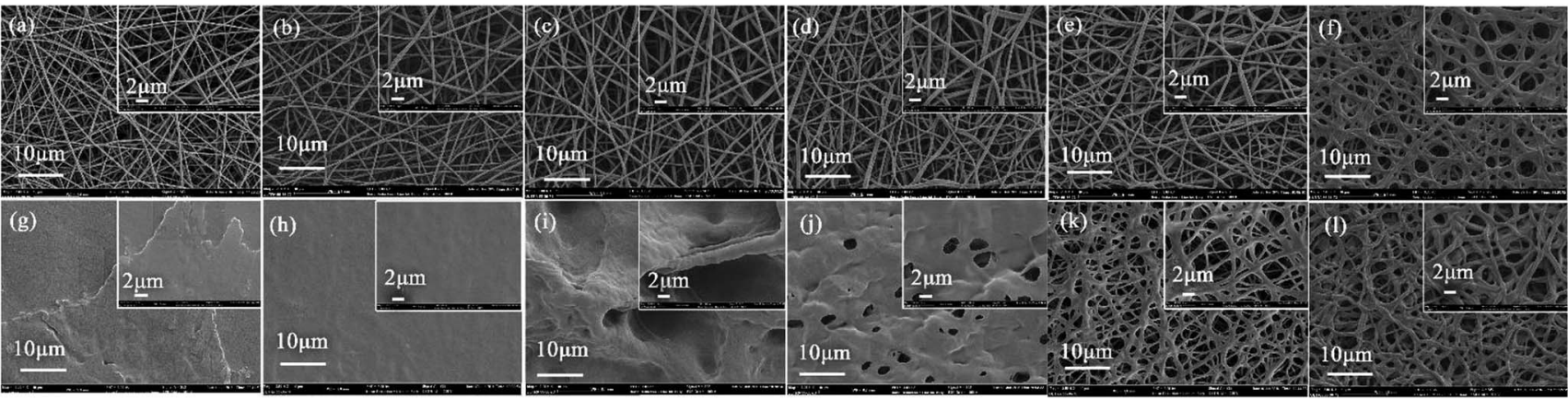

Fig. 12 SEM images of the crosslinked PVA nanofibres at different crosslinking conditions, (a) un-crosslinked; (b) GA 50\% (v/v), crosslinked at $30{ }^{\circ} \mathrm{C}$ for $24 \mathrm{~h}$; (c) GA $25 \%$ (v/v), crosslinked at $60{ }^{\circ} \mathrm{C}$ for $24 \mathrm{~h}$; (d) GA $25 \%$ (v/v), crosslinked at $30{ }^{\circ} \mathrm{C}$ for $24 \mathrm{~h}$; (e) GA $25 \%$ (v/v), crosslinked at $30{ }^{\circ} \mathrm{C}$ for $48 \mathrm{~h}$; (f) GA $25 \%$ (v/v), crosslinked at $30{ }^{\circ} \mathrm{C}$ for $72 \mathrm{~h}$. (g)-(l) The corresponding samples immersed in water for $48 \mathrm{~h}$.

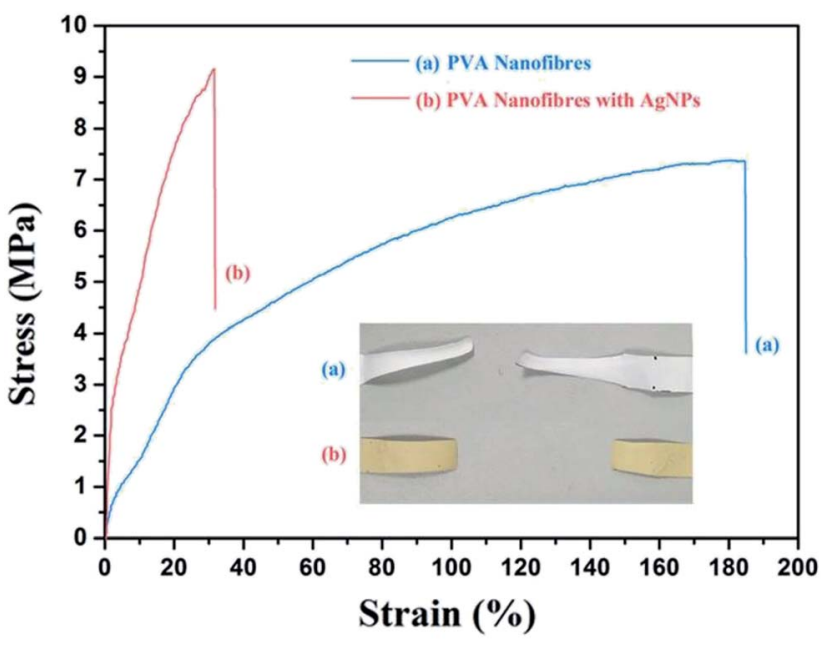

Fig. 13 The stress-strain curves of neat PVA and PVA/Ag NP nanofibre mats.

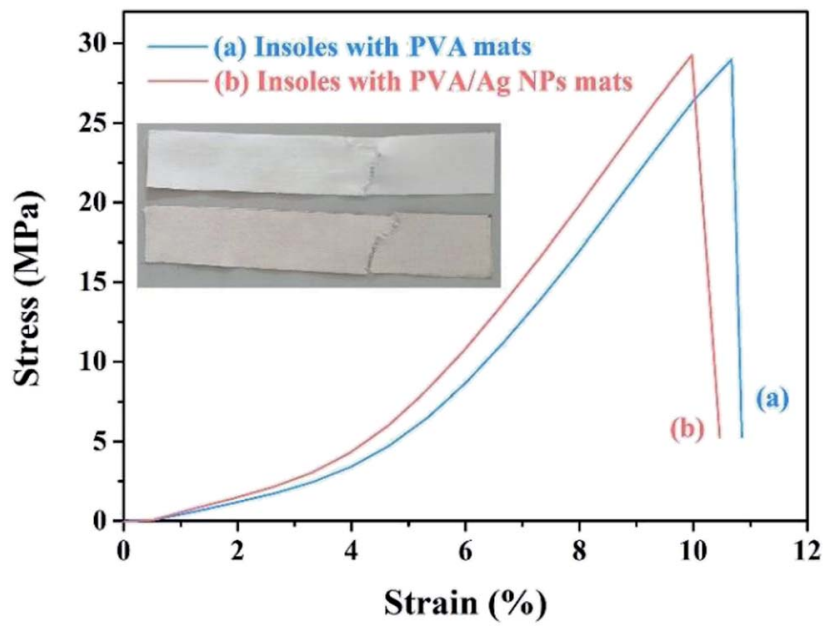

Fig. 14 The stress-strain curves of insoles inlayed with neat PVA nanofibre mats and insoles inlayed with PVA/Ag NP nanofibre mats.
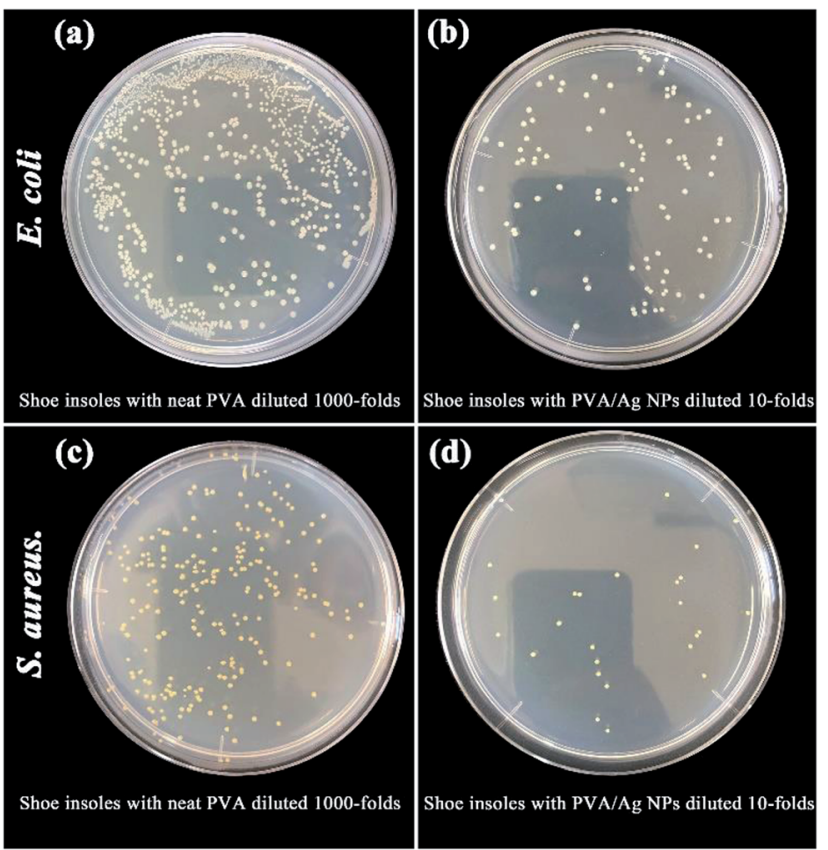

Fig. 15 The antibacterial activity of insoles against Gram-negative bacteria E. coli and Gram-positive bacteria S. aureus.

interplanar spacing is about $0.23 \mathrm{~nm}$, corresponding to the $\mathrm{Ag}$ (1 11 1) planes. $^{30}$

In our previous reports, we found that a chelating effect between the $\mathrm{Ag}^{+}$ions and hydroxyl groups in PVA. The high viscosity of the PVA solution restricts the Ag crystal growth rate and Ag NP aggregation. ${ }^{11}$ We also studied the effectiveness of PVA concentration and reductant dosage on the sizes of $\mathrm{Ag}$ NPs. ${ }^{15}$ The PVA/Ag NP nanofibres with different sizes of Ag NPs are shown in Fig. 10. With an increasing mass fraction of PVA from $10 \mathrm{wt} \%$ to $12 \mathrm{wt} \%$, the average diameters of $\mathrm{Ag}$ NPs decreased slightly, which means that the viscosity has a negligible effect on size control. However, with increasing $\mathrm{AgNO}_{3}$, the average diameters of the Ag NPs decreased from $1.53 \mathrm{~nm}$ to $1.41 \mathrm{~nm}$; with increasing TP, the average diameters of Ag NPs increased from $1.53 \mathrm{~nm}$ to $2.99 \mathrm{~nm}$, suggesting a significant effect of the reductant dosage on size control. The solution transparency declined with increasing Ag NP diameter. The 
Fig. 10B inset exhibits the corresponding diluted PVA/Ag NP solutions, in which the solution with $4 \mathrm{wt} \% \mathrm{AgNO}_{3}$ shows the highest transparency, suggesting the smallest particle diameter. As shown in Fig. 11, the early generations of $\mathrm{Ag}$ atoms are just like starter seeds. The addition of TP leads to a continuous supply of Ag atoms, which add to the surface of the seeds. As a result, the nuclei will grow into nanocrystals of increasingly larger size. Besides growth via atomic addition, the nuclei and nanocrystals can directly merge into larger objects via agglomeration. This result was also confirmed by corresponding UV-vis spectra (see Fig. 10B).

In order to obtain a PVA/Ag NP fibrous membrane with porous structures and water stability, we used GA vapour as the cross-linking agent to treat it, and investigated the effects of cross-linking conditions. As displayed in Fig. 12, treating with high concentrations GA vapour (i.e. $50 \mathrm{v} / \mathrm{v}$ ) or at a high temperature (i.e. $60{ }^{\circ} \mathrm{C}$ ) for $48 \mathrm{~h}$, the nanofibre mats swelled severely after soaking. However, after the treatment with GA vapour (i.e. $25 \mathrm{v} / \mathrm{v}$ ) at $30{ }^{\circ} \mathrm{C}$ for $24 \mathrm{~h}$, the nanofibre mats partially dissolved in water after being immersed in water for $48 \mathrm{~h}$, suggesting insufficient crosslinking. Furthermore, the sample after crosslinking for $48 \mathrm{~h}$ became insoluble, and still maintained excellent porous fibre structures even after immersion in water for $48 \mathrm{~h}$. However, the sample was excessive crosslinked after treatment with GA vapor (i.e. $25 \mathrm{v} / \mathrm{v}$ ) at $30{ }^{\circ} \mathrm{C}$ for $72 \mathrm{~h}$.

Mechanical properties can be considered as one of the effective factors in the application. The increase of solution conductivity leads to strong electrically driven stretching forces during the electrospinning, which will benefit the orientation and stretching of the chains along the fibre axis. ${ }^{31-33}$ The membranes exhibited increased tensile strength from $7.31 \pm$ 0.54 to $9.19 \pm 0.83 \mathrm{MPa}$ with the formation of Ag NPs, which can be caused by orientation of the macromolecule in the fibres. However, the final elongation at the breakage of PVA/Ag NP membranes decreased greatly, compared with the neat PVA fibrous membranes, which is shown in Fig. 13. It is indicated that due to the very high surface area and aggregation tendency of $\mathrm{Ag}$ NPs, the applied stress is expected to be easily transformed from the matrix onto the $\mathrm{Ag}$ NPs, leading to stress concentration. Also, the Ag NPs will block the movement of macromolecular chains and improve the stiffness and strength. ${ }^{34-36}$ Membrane deformation behaviour images are shown in the Fig. 13 inset. What's more, the tensile stresses of the shoe insoles with a neat PVA nanofibrous membrane and shoe insoles with a PVA/Ag NP nanofibrous membrane show no significant difference (see Fig. 14), suggesting the woven cotton as the principal in the insoles plays a leading role in the mechanical properties.

The antimicrobial activity was quantitatively evaluated using E. coli and $S$. aureus, as shown in Fig. 15. Even though the bacteria solution with a shoe insole containing neat PVA has been diluted 1000-fold, the colony count is still larger than that of the bacteria solution, with the shoe insole containing PVA/Ag NPs being diluted 10-fold. The antibacterial rates of the shoe insole with Ag NPs against both $E$. coli and $S$. aureus were greater than $99 \%$. As we know, Ag NPs can attach to the surface of the bacteria cell membrane, disturbing the respiration functions of the bacteria cell to achieve antibacterial action. ${ }^{37}$ Also, Ag NPs of small sizes (i.e. 1-10 nm) may serve as a vehicle to deliver $\mathrm{Ag}^{+}$ more effectively to the bacteria cytoplasm and membrane. ${ }^{38,39}$ Thus, small size Ag NPs with a larger surface area may have a higher antibacterial activity than larger ones.

\section{Conclusions}

In summary, we showed a green and facile synthesis of well dispersed small Ag NPs embedded in electrospun PVA nanofibres by applying a combination of electrospinning and a green reduction approach. Significantly, the effect of the solution properties on the fibre morphology and Ag NPs diameter were thoroughly investigated. Given the solubility of PVA, the prepared nanofibrous membrane was post-treated with GA in the vapour phase, and the optimized condition was determined (i.e. GA $25 \mathrm{v} / \mathrm{v}, 30{ }^{\circ} \mathrm{C}$ for $48 \mathrm{~h}$ ). Furthermore, hybrid nanofibre fabrics with a PVA/Ag NP nanofibrous membrane layered on the substrates were developed and applied to shoe insoles and exhibited a superior antimicrobial activity, benefiting from the existence of Ag NPs. Additionally, the addition of a PVA/Ag NP nanofibrous membrane has no impact on the mechanical performance of shoe insoles. These properties suggest that the electrospun composite nanofibre fabrics containing green reduced Ag NPs could be an appropriate candidate for antibacterial insoles. Also, this manner opens the door for diverse possibilities in the preparation of nanomaterials integrated into textiles with desired functionalities.

\section{Conflicts of interest}

There are no conflicts to declare.

\section{Acknowledgements}

This study is financially supported by the Natural Science Foundation of Zhejiang Province (LQ19E030021), the Zhejiang Sci-Tech University Scientific Research Foundation (17072190Y), the Special Foundation for Excellent Doctor of Zhejiang top priority discipline of textile science and engineering of the priority discipline (2017YBZX13), and the Key Laboratory of Advanced Textile Materials and Manufacturing Technology (Zhejiang Sci-Tech University), Ministry of Education (2017QN09). Meanwhile, this work is also funded by the 2011 Collaborative Innovation Center for Garment Personalized Customization of Zhejiang Province.

\section{References}

1 Y. Gao and R. Cranston, Text. Res. J., 2008, 78, 60-72.

2 H. Li, J. Zhou, R. Shi and W. Y. Chen, Afr. J. Biotechnol., 2011, 10, 14493-14497.

3 I. P. Fernandes, J. S. Amaral, V. Pinto, M. J. Ferreira and M. F. Barreiro, Carbohydr. Polym., 2013, 98, 1229-1235.

4 K. Lee and S. S. Lee, Text. Res. J., 2015, 85, 1999-2008.

5 S. Chernousova and M. Epple, Angew. Chem., Int. Ed. Engl., 2013, 52, 1636-1653. 
6 P. Velmurugan, M. Cho, S. S. Lim, S. K. Seo, H. Myung, K. S. Bang, S. Sivakumar, K. M. Cho and B. T. Oh, Mater. Lett., 2015, 138, 272-275.

7 K. Loza and M. Epple, RSC Adv., 2018, 8, 24386-24391.

8 W. J. Jin, H. J. Jeon, J. H. Kim and J. H. Youk, Synth. Met., 2007, 157, 454-459.

9 P. Rauwel, S. Küünal, S. Ferdov and E. Rauwel, Adv. Mater. Sci. Eng., 2015, 2015, 1-9.

10 R. D. Rivera-Rangel, M. P. González-Muñoz, M. AvilaRodriguez, T. A. Razo-Lazcano and C. Solans, Colloids Surf., A, 2018, 536, 60-67.

11 L. Du, H. Z. Xu, T. Li, Y. Zhang and F. Y. Zou, Fibers Polym., 2016, 17, 1995-2005.

12 Y. Wang, Z. X. Shi and J. Yin, ACS Appl. Mater. Interfaces, 2011, 3, 1127-1133.

13 P. Wang, H. Zhu, S. Y. Bao, M. L. Du and M. Zhang, J. Phys. D: Appl. Phys., 2013, 46, 345303-345310.

14 Y. B. Zhang, L. Ye, M. Cui, B. G. Yang, J. J. Li, H. Sun and F. L. Yao, RSC Adv., 2015, 5, 78180-78191.

15 L. Du, H. Z. Xu, T. Li, Y. Zhang and F. Y. Zou, RSC Adv., 2017, 7, 31310-31318.

16 Y. S. Pan, J. Ding, Y. Chen and Q. Q. Shen, Mater. Technol., 2016, 31, 266-273.

17 G. Yang, X. L. Li, Y. He, J. K. Ma, G. L. Ni and S. B. Zhou, Prog. Polym. Sci., 2018, 81, 80-113.

18 J. J. Xue, J. W. Xie, W. Y. Liu and Y. N. Xia, Acc. Chem. Res., 2017, 50, 1976-1987.

19 G. X. Zhao, X. H. Zhang, T. J. Lu and F. Xu, Adv. Funct. Mater., 2015, 25, 5726-5738.

20 A. K. Yetisen, H. Qu, A. Manbachi, H. Butt, M. R. Dokmeci, J. P. Hinestroza, M. Skorobogatiy, A. Khademhosseini and S. H. Yun, ACS Nano, 2016, 10, 3042-3068.

21 Y. Xu, J. L. Sheng, X. Yin, J. Y. Yu and B. Ding, J. Colloid Interface Sci., 2017, 508, 508-516.

22 A. A. Babar, D. Y. Miao, N. Ali, J. Zhao, X. F. Wang, J. Y. Yu and B. Ding, ACS Appl. Mater. Interfaces, 2018, 10, 2286622875 .
23 A. A. Oun and J. W. Rhim, Carbohydr. Polym., 2017, 169, 467479.

24 G. N. Al'tshuler and E. V. Ostapova, Russ. J. Phys. Chem. A, 2009, 83, 1032-1035.

25 H. Zhu, M. L. Du, M. L. Zou, C. S. Xu, N. Li and F. Y. Fu, J. Mater. Chem., 2012, 22, 9301-9307.

26 R. J. Liao, Z. H. Tang, Y. D. Lei and B. C. Guo, J. Phys. Chem. C, 2011, 115, 20740-20746.

27 M. L. Hallensleben, Ullmann's Encyclopedia of Industrial Chemistry, Wiley, Hannover, 2000.

28 L. Du, H. Z. Xu, Y. Zhang and F. Y. Zou, Fibers Polym., 2016, 17, 751-759.

29 Z. L. Xu, K. Miyazaki and T. Hori, Appl. Surf. Sci., 2016, 370, 243-251.

30 W. Zhang, W. Wu, Y. Long, F. S. Wang and J. T. Ma, J. Colloid Interface Sci., 2018, 522, 217-227.

31 L. Gong, D. B. Chase, I. Noda, J. L. Liu, D. C. Martin, C. Y. Ni and J. F. Rabolt, Macromolecules, 2015, 48, 6197-6205.

32 W. A. Yee, M. Kotaki, Y. Liu and X. H. Lu, Polymer, 2007, 48, 512-521.

33 J. T. Gu, H. H. Gu, J. Cao, S. J. Chen, N. Li and J. Xiong, Appl. Surf. Sci., 2018, 439, 589-597.

34 T. Ghaffari and F. Hamedi-Rad, J. Dent. Res. Dent. Clin. Dent. Prospects, 2015, 9, 40-43.

35 A. Sodagar, M. Z. Kassaee, A. Akhavan, N. Javadi, S. Arab and M. J. Kharazifard, J. Prosthodont. Res., 2012, 56, 120-124.

36 J. Z. Liang, D. R. Duan, C. Y. Tang, C. P. Tsui and D. Z. Chen, Polym. Test., 2013, 32, 617-621.

37 G. Franci, A. Falanga, S. Galdiero, L. Palomba, M. Rai, G. Morelli and M. Galdiero, Molecules, 2015, 20, 8856-8874.

38 L. D. Tijing, M. T. G. Ruelo, A. Amarjargal, H. R. Pant, C. H. Park and C. S. Kim, Mater. Chem. Phys., 2012, 134, 557-561.

39 Z. M. Xiu, Q. B. Zhang, H. L. Puppala, V. L. Colvin and P. J. J. Alvarez, Nano Lett., 2012, 12, 4271-4275. 